\title{
RELACIONES ENTRE DIMENSIONES DE APEGO ADULTO Y DIMENSIONES DE IRA EN ADULTOS JÓVENES PERUANOS
}

\section{Giovana Violeta Chanllío Ríos}

Psicóloga Clínica y Psicoterapeuta, Salud Mental para Todos, Lima, Perú

Correspondencia: giovana.chanllio@gmail.com

Artículo basado en la Tesis de pregrado Relaciones entre dimensiones de apego adulto y de ira en adultos jóvenes, realizada en el 2015, supervisada por Ana Mercedes Caro Cárdenas y revisada por Magaly Suzy Noblega Mayorga en la Pontificia Universidad Católica del Perú.

\section{Resumen}

La presente investigación tuvo como objetivo principal describir las relaciones entre las dimensiones de apego adulto (Ansiedad y Evitación) y las de ira (Ira-Rasgo, Ira-Estado, y Expresión de Ira, esta última con sus sub-dimensiones Ira Contenida, Ira Manifiesta, Control de la Ira Suprimida y Control de la Ira Manifiesta) en jóvenes adultos peruanos mediante una innovadora herramienta virtual de recolección de datos.

Para recolectar los datos, se realizó la aplicación virtual de cuestionarios de autorreporte (ECR-S y MLSTAXI) en 112 adultos jóvenes peruanos. Los datos se procesaron mediante análisis estadísticos no paramétricos.

En este estudio, se encontró relaciones entre la dimensión de Ansiedad con las dimensiones de Ira: Ira-Rasgo $\left(r_{\mathrm{s}}=.32\right)$, Ira Estado $\left(\mathrm{r}_{\mathrm{s}}=.20\right)$, Expresión de la Ira $\left(\mathrm{r}_{\mathrm{s}}=-.21\right)$, Control de la Ira Manifiesta $\left(\mathrm{r}_{\mathrm{s}}=.19\right)$, y Control de la Ira Suprimida $\left(\mathrm{r}_{\mathrm{s}}=.20\right)$.

Los resultados, de ser replicados, podrían ser útiles para los terapeutas trabajando con personas con altos niveles de la dimensión de Ansiedad de apego. En específico, entender cómo esta se relaciona con la Ira podría derivar en el desarrollo de estrategias que resulten más efectivas para la expresión de dicha emoción.

Palabras clave: Apego, Ira, Ansiedad, Evitación, Apego Adulto

\section{RELATIONS BETWEEN ATTACHMENT AND ANGER DIMENSIONS IN PERUVIAN YOUNG ADULTS}

\begin{abstract}
The present study assessed the associations between the dimensions of Adult Attachment: Avoidance and Anxiety, and Anger components: Trait Anger, State Anger, and Anger Expression (with its sub-dimensions of Anger Out, Anger In, Anger-Out Control, and Anger-In Control). Computer-based self-report measures were used (ECR-S and ML-STAXI) for data collection, and non-parametric analysis were used to find the correlations.

At a descriptive level, the data states that Anxious participants had higher State Anger $(\mathrm{rs}=.20)$, lower Anger Expression ( $\mathrm{rs}=-.21$ ), higher Anger-Out Control ( $\mathrm{rs}=.19)$ and higher Anger-In Control ( $\mathrm{rs}=.20)$.

These results, if replicated, could be informative to therapists working individuals with high levels of attachment-oriented Anxiety, as a better understanding of this could be used to develop more effective interventions for expressing their anger.
\end{abstract}

Keywords: Attachment, Anger, Trait-Anger, Attachment-related avoidance, Attachment-related anxiety

\section{Introducción}

\subsection{Apego Adulto}

Según Bowlby (1982), los seres humanos están predispuestos a desarrollar lazos emocionalmente íntimos y perpetuos con los cuidadores adultos (Hazan y Selcuk, 2015) para obtener protección ante amenazas y una sensación de seguridad (Feeney y Collins, 2001). Una de las situaciones que representa mayor amenaza es el hecho o posibilidad de que cuando la figura de apego no está cerca, accesible ni atenta (Fraley, 2010).

El vínculo de apego formado con el cuidador adulto brinda información a la persona acerca del 
grado en que es merecedor de afecto y atención, influye en su confianza hacia los demás, crea necesidades afectivas, y establece expectativas de próximos vínculos. Se convierte así en un modelo para las relaciones futuras (Siegel, 2012, Stevens, 2014), sobre todo con figuras significativas en la adultez (Hazan y Shaver, 1987), espeecialmente con la pareja romántica (Fraley, 2010). El vínculo de apego puede predecir las cogniciones, afectos, estrategias de regulación emocional, conductas, y expectativas de las relaciones interpersonales durante toda la vida. (Hazan y Selcuk, 2015; Günaydin, y Shoda, 2015; Sheinbaum et al., 2015; Zayas, Adams y McWilliams, 2015; Bartz, Baldwin, y Lydon, 2015; Fraley, 2010).

Para Fraley (2010), el paralelismo entre el apego infantil y el apego adulto significa que: (a) Las diferencias individuales se manifiestan tanto con el cuidador como con la pareja; (b) Las necesidades de apego (e.g., intimidad emocional, soporte) durante la niñez son similares a las de la adultez, por lo que la pareja se elige en función a la capacidad percibida que tendría para suplirlas; (c) La orientación de apego adulto no es un reflejo exacto de la de la niñez, ya que se incorporan cambios temporales o permanentes dependiendo del contexto y experiencias relevantes de apego en las relaciones cercanas (Mikulincer y Shaver, 2011; Konishi y Hymel, 2014; Feeney y Collins, 2001; Mikulincer, 2000; Adams y McWilliams, 2015; Mikulincer, 1998).

Barthelemew (1990, en Stevens, 2014) propone dos dimensiones básicas para entender los estilos de apego: Ansiedad y Evitación. Cuando una persona evidencia un nivel alto de una o ambas dimensiones, presenta un estilo de apego Inseguro (Diamond, 2015; Fraley, 2010), mientras que si evidencia bajos niveles en ambas dimensiones presenta un estilo de apego Seguro (Diamond, 2015; Bartz, Baldwin, y Lydon, 2015; Zayas, Günaydin, y Shoda, 2015).

Según Diamond (2015), Stevens (2014), Mikulincer y Shaver (2011), Overall, Simpson y Struthers (2013), Loinaz y Echeburúa (2012), Ognibene y Collins (1998), y Alonso-Arbiol, Balluerka, y Shaver (2007), las dimensiones se definen de la siguiente manera:

1.1.1 Dimensión de Ansiedad. Referente a si la persona cree merecer amor, y al grado en que se preocupa de ser rechazada por los demás.

Las personas que obtienen altos puntajes en esta dimensión se caracterizan por demandar una alta intimidad emocional. Creen, además, que los demás no tienen interés en mantener el vínculo con ellas, temen al rechazo de las personas significativas para ellos, tienen una percepción negativa de sí mismos, y mantienen una percepción positiva de los demás.

Durante la relación, pueden adoptar una actitud hipervigilante hacia posibles situaciones de abandono, entendidas como la posibilidad de que su pareja no desee mantener el vínculo. Ante conflictos de pareja, tienden a intensificar el estrés, tener pensamientos rumiativos, y maximizar la vivencia de ira.

1.1.2 Dimensión de Evitación. Se refiere al grado en que la persona percibe que los demás son responsivos, y también al grado en el que se encuentra cómoda con la intimidad y dependencia hacia los demás.

Las personas con altos niveles de esta dimensión se caracterizan por sentirse incómodas ante la cercanía emocional. Además, suelen tener una percepción positiva de sí mismos y negativa de los demás pues perciben que se les demanda mayor intimidad que la que ellos se sienten cómodos dando. Asimismo, suelen desconfiar del otro, por lo que no se permiten depender de personas cercanas.

Durante la relación, estas personas priorizan su independencia y otras esferas de su vida por sobre el vínculo romántico. Ante conflictos de pareja, se les dificulta identificar sus emociones y tienden a redirigir su atención lejos del conflicto. Así, pueden realizar acciones impulsivas. Además, evitan buscar soporte por parte de los demás para solucionar el problema; en cambio, se distancian de la pareja.

Para Bélanger y Brisebois (2010), la amenaza de pérdida del vínculo o la anticipación de esta son interpretadas como abandono, rechazo, trasgresión de los límites personales, o vulnerabilidad. Ante ello, las personas suelen reaccionar con ira o miedo (Moscoso, 2000), siendo la ira la emoción predominante en este tipo de conflictos (Izard, 1991; Muro, 2010). 


\subsection{Ira}

En la definición de esta emoción, confluyen factores fisiológicos, fenomenológicos, cognitivos, y conductuales que componen la vivencia individual (Moscoso, 2000; Genest y Mathieu, 2014). Moscoso (2000) propone tres dimensiones:

\subsubsection{Ira-Rasgo. Disposición biológica y} constante de la persona a experimentar sentimientos de ira. Personas con mayores puntajes en esta dimensión tienden a: reaccionar de manera iracunda ante situaciones de dificultad, experimentar ira con una intensidad elevada, y sentir ira con frecuencia (Muro, 2011).

1.2.2 Ira-Estado. Emoción transitoria y variable en intensidad (desde un fastidio hasta rabia).

1.2.3 Expresión de ira. Manera de reaccionar cuando se experimenta ira. Expresada de manera asertiva, es adaptativa para las relaciones interpersonales. Lo contrario, implica una activación fisiológica que desemboca en dificultades en la salud física y mental (Spielberger y Reheiser, 2009), además de múltiples consecuencias en el mantenimiento de vínculos.

1.2.3.1 Ira Manifiesta. Expresión abierta de ira y la frecuencia con que se expresa hacia otras personas $\mathrm{u}$ objetos. En esta dimensión también se incluye las conductas agresivas -como destrucción de objetos o asaltos a otras personas.

\subsubsection{Control de la Ira Manifiesta. Control} de la expresión abierta de la ira mediante estrategias y acciones concretas.

1.2.3.3 Ira Contenida. Frecuencia con que un individuo suprime la ira para redirigirla a sí mismo (Spielberger, 1985), o para procesarla a nivel cognitivo y emocional.

\subsubsection{Control de la Ira Contenida. Control de la vivencia de la ira.}

\subsection{Relación entre Apego e Ira}

En personas con apego Seguro, la expresión de la ira en las relaciones de pareja sirve para disuadir a la figura de apego de alejarse, comunicar una reacción a un mal trato desmerecido, y reparar la relación (Mikulincer y Shaver, 2011). Expresar la "ira con esperanza" (Mikulincer y Shaver, 2011) suele motivar a la pareja a prestar más atención al sujeto.

En cambio, las personas con apego Inseguro suelen reaccionar mediante la "ira con desamparo" (Mikulincer y Shaver, 2011), la cual puede dañar a la pareja con pensamientos y/o actos agresivos (Bowlby, 1973). Esta ira disfuncional en las relaciones cercanas, ocurriría, según Dutton (2008, en Genest y Mathieu, 2014), debido a una activación inadecuada del sistema de apego.

Diversos estudios evidencian la relación entre las dimensiones de Apego y las de Ira en la adultez. Las personas con predominancia de las dimensiones de Ansiedad y Evitación muestran mayores niveles de ira y hostilidad que aquellas con apego seguro (Konishi y Hymel; 2014) en las amistades cercanas (Muris, Meesters, Morren, y Moorman, 2004) y con los compañeros de clases en la universidad (Zimmermann, 2004; Kerns y Stevens, 1996, en Konishi y Hymel, 2014).

\section{Ansiedad e Ira}

Las personas con predominancia de la dimensión Ansiedad suelen experimentar ira con mayor frecuencia que aquellos con predominancia de Evitación (Mikulincer y Shaver, 2007), sobre todo cuando sienten que la pareja no está disponible y que el apoyo que les brindó no es suficiente (Overall, Simpson, y Struthers, 2013). Sin embargo, la dependencia hacia la pareja hace que eviten expresar la ira, redirigiéndola hacia sí mismos. Por lo tanto, tienden a expresar su ira solo cuando las estrategias de manipulación sobre el otro fallan (Follingstad, Bradley, Helff, y Laughlin, 2002). Esto se traduce en resentimiento, autocrítica, tristeza, pensamientos rumiativos (Gormley y McNiel, 2010; Mikulincer y Shaver, 2011; Loinaz y Echeburúa, 2012), buscar la proximidad como protección, y en volverse sumamente emocionales y controladores para recuperar la atención de la pareja (Stevens, 2014; Mikulincer, Shaver y Pereg; 2003, Mikulincer y Shaver, 2011).

Cuando anticipan que el rechazo o abandono de la pareja podría darse, sienten un alto nivel de angustia (Follingstad, Bradley, Helff, y Laughlin, 2002), lo cual podría desembocar en violencia (Konishi y Hymel, 2014) y conductas impulsivas para evitar el abandono (Gormley y McNiel, 2010; Mikulincer y Shaver, 2011; Loinaz y Echeburúa, 2012).

Diversos estudios han encontrado que los hombres coléricos y con predominancia de la 
dimensión de Ansiedad experimentan extremos niveles de ira ante situaciones que implican abandono (Dutton y Golant, 1995; Genest y Mathieu, 2014), suelen expresar su ira mediante la agresión, y aceptan las conductas agresivas por parte de sus parejas (Diamond y Hicks, 2005; en Mikulincer y Shaver, 2011).

\section{Evitación e Ira}

Las personas con predominancia de la dimensión Evitación favorecen la supresión de la ira (Mikulincer y Shaver, 2011), y cuando la expresan, lo hacen de manera indirecta y en forma de hostilidad inespecífica o actitudes de odio (Nisenbaum y Lopez, 2015). Esto se debe a que el expresar la ira implica para ellos involucrarse demasiado en la relación, incongruente con su necesidad de distanciarse y ser independientes (Nisenbaum y Lopez, 2015). Si expresan su ira, lo suelen hacer durante una discusión de pareja donde se le pide un mayor nivel de compromiso (Genest y Mathieu, 2014), pues lo entienden como un intento de intrusión y un bloqueo a su autonomía (Keltner y Lerner, 2010).

Otras estrategias usadas ante conflictos de pareja son: negar sus necesidades de apego, evitar la discusión del problema o darle menos importancia, las cuales solo exacerban su estado de ira (Overall, Simpson, y Struthers, 2013; Gormley y McNiel, 2010).

Estas personas suelen presentar un déficit en reconocer las propias emociones, que responde a la búsqueda de mantener las emociones bajo control y evitar experimentar las emociones propias del abandono (Mikulincer y Shaver, 2011). Incluso, pueden presentar altos niveles de ira y hostilidad cuando deben describir sus necesidades afectivas o sus emociones (Rholes, Simpson y Oriña, 1999). Generalmente, reportan no sentir intensos niveles de ira, a pesar de estar presentando una elevada reacción fisiológica ante eventos que los molestan durante el contexto de evaluación (Mikulincer y Shaver, 2011).

La importancia del tema de la presente investigación radica, principalmente, en que tiene incidencia en diversos ámbitos del desarrollo del ser humano: sus relaciones interpersonales y su regulación emocional. Actualmente, no se puede hablar de una predominancia de estilo de apego en un contexto latinoamericano, lo cual sí ocurre en un contexto norteamericano (Hazan y Shaver, 1987). Asimismo, las investigaciones latinoamericanas con respecto a la medición o expresión de la ira se han visto limitadas por la inaccesibilidad de instrumentos, principalmente por la ausencia de validaciones en nuestro contexto.

Por otro lado, el estilo Inseguro de apego en adultos jóvenes es un factor de riesgo para conductas como la violencia, abuso sexual, poco control de impulsos, conductas sexuales de riesgo, y abuso emocional (Oshri, Sutton, Clay-Warner, y Miller, 2015). Si bien estas conductas no son una consecuencia directa de la dimensión predominante de apego, sí son un producto de la intensa ira acumulada debido a la frustración en suplir las necesidades de apego (Grych y Kinsfogel, 2010; Bélanger y Brisebois, 2010).

Con respecto a la ira, esta por sí misma no ocasiona daños en los demás, pero sí su expresión mediante violencia psicológica o verbal. Esto se da en el $69 \%$ de parejas peruanas, cuyas víctimas son, sobre todo, mujeres entre 15 a 29 años de edad (Secretaría Nacional de la Juventud, 2015).

Es de interés, entonces, empezar a prevenir y solucionar estos temas desde una perspectiva psicológica, sobre todo en un contexto como el peruano donde: el $34,1 \%$ de los adultos jóvenes peruanos se encuentra recluido en un penal (INPE, 2015), el 72,4\% de peruanas ha sufrido violencia por parte de su esposo o compañero (INEI, 2014), se ha realizado la mayor cantidad de abusos sexuales de Latinoamérica (Mujica, 2011), y solamente el $74 \%$ de mujeres usa algún método anticonceptivo (INEI, 2014).

Asimismo, es de interés notar la relación entre la dimensión ansiosa de apego con conductas autolesivas o heteroagresivas para evitar el abandono (Loinaz y Echeburúa, 2012). Según un estudio realizado por Rospigliosi (2010), el 27\% de adolescentes peruanos alguna vez se ha autolesionado.

Actualmente, hay estudios que relacionan los estilos de apego adulto con variables como la empatía en parejas (Guzmán y Trabucco, 2014) y la violencia hacia la pareja (Loinaz y Echeburúa, 2012), pero aún no se ha realizado -a la fecha del estudio- alguna investigación latinoamericana que relacione los constructos de apego e ira. Por ello, el presente estudio representa una exploración inicial de las relaciones entre las dimensiones de Apego y las dimensiones de Ira en jóvenes adultos peruanos. 


\section{Método}

\subsection{Participantes}

El estudio incluyó 112 participantes con edades entre 19 a 29 años (64.3\% mujeres; $34.8 \%$ entre 22 y 23 años; 96\% residencia en Lima, 36.6\% con estudios técnicos superiores). La mayor parte de ellos $(80.4 \%)$ reportó resultados en función a su relación actual de pareja, con una duración promedio de 1 a 3 años (58.3\%). Quienes reportaron una relación pasada, se refirieron principalmente a relaciones con una duración de 2 a 4 años (81.8\%).

La mayor parte de los participantes reportó un vínculo de Enamorados-No convivientes (78.6\%) $\mathrm{y}$ encontrarse muy satisfechos (53.57\%) o satisfechos (33\%) con dicho vínculo.

Los criterios de inclusión fueron: (a) adultos jóvenes entre 19 a 29 años y (b) mantener una relación romántica monogámica y heterosexual con un mínimo de 6 meses de duración al momento del estudio, o haber tenido una relación de al menos 2 años de duración. El criterio de exclusión fue: no aceptar el consentimiento informado. Todos los participantes firmaron, virtualmente y con un seudónimo, un Consentimiento Informado.

Ninguno de los protocolos fue eliminado dado que la herramienta de recolección de datos se configuró para contabilizar, de entre 170 personas que accedieron a los cuestionarios, a los 112 protocolos que se habían culminado en su totalidad.

\subsection{Medición}

\subsubsection{Cuestionario de Relaciones Interpersonales} - ECR-S (Experiences in Close Relationships, Spanish version; Alonso-Arbiol, Balluerka, y Shaver, 2007). Este es un instrumento autoadministrado que evalúa las dos dimensiones de Apego Adulto: Ansiedad y Evitación con 36 ítemes divididos de manera equitativa para cada dimensión. Brinda siete opciones de respuesta entre totalmente en desacuerdo (1) y totalmente de acuerdo (7), configuradas en un formato de respuesta tipo Likert. El puntaje de cada dimensión se calcula obteniendo la media de los puntajes.

El ECR-S tiene altas consistencia interna $(\alpha=.85$ para Ansiedad, $\alpha=.87$ para Evitación), confiabilidad test-retest (Alpha de Cronbach de .86 para Ansiedad y .87 para Evitación), y validez convergente con otros cuestionarios como Love
Scale (Carreño y Serrano, 1995) y Marital Satisfaction Inventory-Revised (Reig-Feller, Cepeda-Benito, y Snyder, 2004). De manera consistente con investigaciones previas, el presente estudio tiene alta consistencia interna ( $\alpha=.84$ para Evitación y $\alpha=.91$ para Ansiedad).

\subsubsection{Inventario Multicultural Latinoamericano de} la Expresión de la Cólera y Hostilidad - MLSTAXI (Moscoso, 2000). Este es un instrumento auto-administrado que evalúa la experiencia, expresión y control de la ira en poblaciones latinoamericanas e hispanohablantes, con 44 ítemes para evaluar las tres dimensiones. Para la dimensión Ira-Estado de 10 ítemes, se brinda una escala Likert de 4 puntos (no en lo absoluto: 1, mucho: 4); para las dimensiones Ira-Rasgo (10 ítemes) y Expresión de la Ira (24 ítemes), se brinda una escala Likert de 4 puntos (nunca: 1, casi siempre: 4). Las dimensiones de Ira-Estado e IraRasgo se calculan mediante una sumatoria de los puntajes (mínimo: 10, máximo: 40), y la dimensión de Expresión de la Ira se calcula mediante la fórmula: Ira Manifiesta + Ira Suprimida - Control +36 (puntaje mínimo obtenible: 0, máximo: 72).

El ML-STAXI tiene alta consistencia interna $(\alpha=$ .82 para Ira-Estado, $\alpha=.83$ para Ira-Rasgo, $\alpha=.70$ para Ira Manifiesta, $\alpha=.65$ para Ira Contenida, $\alpha=$ .70 para Control de Ira Manifiesta y $\alpha=.86$ para Control de Ira Contenida). De manera consistente con investigaciones previas, en el presente estudio la escala muestra buenos niveles de confiabilidad (Ira-Estado $\alpha=.83$, Ira-Rasgo $\alpha=.82$, Expresión de Ira $\alpha=.85$, Ira Manifiesta $\alpha=.67$, Ira Suprimida $\alpha=.77$, Control de Ira Manifiesta $\alpha=.84$, y Control de Ira Suprimida $\alpha=.93$ ).

\subsection{Procedimiento}

Las pruebas se presentaron en formato virtual (previa autorización expresa de los autores) mediante Qualtrics, software especializado en la recolección y análisis de datos virtuales con fines académicos. Para lograr que la herramienta virtual sea una verdadera ventaja en la recolección de datos, se realizó las siguientes configuraciones:

(a) para evitar efectos de resistencia o deseabilidad social: bloquear la opción de retroceder a páginas anteriores o modificar respuestas pasadas cuando se volvían a presentar los ítemes refraseados en el transcurso de la evaluación; 
(b) para evitar omisiones: mostrar mensajes de error en las respuestas incompletas y resaltar con color rojo las preguntas faltantes;

(c) para propiciar que los participantes lean los ítemes con detenimiento: agregar una barra de proceso ( $0 \%$ completado $-100 \%$ completado) y brindar la opción de culminar el cuestionario en un lapso de días estipulado sin perder las respuestas ya marcadas; $y$

(d) para facilitar la comprensión y claridad de las indicaciones generales de los cuestionarios: seguir las indicaciones de diversos autores (e.g., Varnhagen et al., 2005; Riva, Teruzzi y Anolli, 2003) de modificar el texto y formato de la herramienta subrayando palabras clave en dichos enunciados (e.g., "indica la frecuencia"), distribuir los ítemes en diferentes páginas, y compartir en todos los pies de página los correos de la investigadora y asesora para cualquier duda con respecto a los ítemes.

Para la difusión de la encuesta, se publicó el link de ingreso en diversas redes sociales, explicitando los criterios de selección en la invitación. Al ingresar a la herramienta virtual, se indagó información en referencia a los criterios de inclusión. Si el participante cumplía con estos criterios, se les mostró preguntas referentes a su relación de pareja: de contar con una relación al momento del estudio, se les preguntó el tipo de relación y la duración de esta; si no era el caso, se les derivó a una ventana donde se pedía reportar la duración de alguna relación romántica pasada. Seguidamente, se mostró el Consentimiento Informado; solamente luego de aceptarlo, pudieron acceder a la Ficha de datos demográficos y los cuestionarios ECR-S y ML-STAXI. La encuesta se cerraba automáticamente con un mensaje de agradecimiento cuando detectaba que el participante no cumplía con una de las etapas de los criterios de inclusión, o si no se aceptó el Consentimiento Informado.

\subsection{Aspectos éticos}

Toda la data recolectada en el presente manuscrito cumplió con los protocolos éticos aprobados por la Pontificia Universidad Católica del Perú. Adicionalmente, se tomó las siguientes medidas de seguridad: (a) no se recolectó datos personales (como e-mail, nombre o dirección); (b) se configuró la herramienta de recolección de datos para no guardar la dirección IP de los participantes; (c) se agregó filtros para evitar que los buscadores (como Google) encuentren la encuesta.

\subsection{Análisis de datos}

Los datos recolectados se exportaron al programa SPSS Statistics 22.0 para describir las variables empleadas y analizar las relaciones que hay entre ellas. Primero, se verificó la confiabilidad de los instrumentos mediante el método de consistencia interna (Alpha de Cronbach). Luego, para obtener mayor información acerca de las variables estudiadas, se ejecutaron análisis exploratorios y se obtuvieron los estadísticos descriptivos. Después, se evaluó la normalidad de la distribución de los puntajes a través de la prueba de normalidad de KolmogorovSmirnov; sin embargo, el nivel crítico fue muy pequeño para las variables $(p<.05)$, por lo que se ejecutó análisis no paramétricos. Luego, se realizaron análisis de correlaciones Spearman entre los puntajes de las dimensiones de Apego y las dimensiones de Ira.

\section{Resultados}

Como se muestra en la tabla 1, se encontró relación entre las variables de Ira y Apego.

\subsection{Relación de Ansiedad e Ira}

Los datos obtenidos brindan soporte empírico a la relación entre la dimensión de Ansiedad y la de Ira-Rasgo, la cual resultó directa y mediana (rs $=.32$ ). Además, se encontró una relación directa y pequeña $(r s=.20)$ con la dimensión de Ira-Estado; una relación inversa y pequeña $(\mathrm{rs}=-.21)$ con la dimensión de Expresión de Ira; una relación directa con Control de Ira Manifiesta (rs =.19); y una relación directa con Control de Ira Suprimida ( $\mathrm{rs}=.20)$.

\subsection{Relación entre Evitación e Ira}

En este estudio no se encontró relación entre la dimensión de Evitación con la variable Ira en ninguna de sus dimensiones. 
Tabla 1

Correlaciones Spearman entre dimensiones de Ira y dimensiones de Apego

\begin{tabular}{|c|c|c|c|c|c|c|c|c|c|}
\hline \multirow[b]{2}{*}{ Dimensiones de Ira } & \multirow[b]{2}{*}{1} & \multirow[b]{2}{*}{2} & \multirow[b]{2}{*}{3} & \multirow[b]{2}{*}{4} & \multirow[b]{2}{*}{5} & \multirow[b]{2}{*}{6} & \multirow[b]{2}{*}{7} & \multicolumn{2}{|c|}{ Dimensiones de Apego } \\
\hline & & & & & & & & Evitación & Ansiedad \\
\hline 1. Ira-Estado & - & & & & & & & .96 & $.20^{*}$ \\
\hline 2. Ira-Rasgo & $.27^{*}$ & - & & & & & & .16 & $.32 *$ \\
\hline 3. Expresión de Ira & -.08 & $-.37^{*}$ & - & & & & & -.13 & $-.21^{*}$ \\
\hline 4. Ira Manifiesta & .11 & $.21^{*}$ & -.05 & - & & & & -.12 & .14 \\
\hline 5. Ira Suprimida & -.00 & -.10 & .16 & -.15 & - & & & -.14 & -.07 \\
\hline 6. Control de Ira Manifiesta & -.00 & $.29^{*}$ & $-.69^{*}$ & $.30^{* *}$ & -.06 & - & & .01 & $.19^{*}$ \\
\hline 7. Control de Ira Suprimida & .12 & $.38^{* *}$ & $-.88^{* *}$ & $.22^{*}$ & .13 & $-.52^{* *}$ & - & .07 & $.20^{*}$ \\
\hline
\end{tabular}

\section{Discusión}

El presente estudio representa una exploración inicial de las relaciones entre las dimensiones de Apego y las dimensiones de Ira en jóvenes adultos peruanos.

\subsection{Relación entre Evitación e Ira}

Una posible explicación para el comportamiento de la variable Evitación podría deberse a que la emoción primaria que estos participantes experimentarían ante una amenaza en las relaciones interpersonales sería el miedo, y no la ira (Diamond, 2015). En este sentido, las investigaciones que se usaron de base para el presente estudio (como Mikulincer y Shaver, 2011) parten del postulado de que la ira es la emoción primaria predominante ante situaciones conflictivas, sobre todo en un contexto de pareja. Dicho postulado surgió, en sus inicios, de las observaciones y mediciones de indicadores fisiológicos, como el aumento de frecuencia cardíaca y sudoración en los participantes (Mikulincer, 1998). Asimismo, postular que era la ira la emoción primaria predominante ante un conflicto de pareja resultaba congruente desde la perspectiva de la Teoría de Apego de Bowlby (1982). No obstante, al interpretar los indicadores fisiológicos como evidencia de una vivencia de ira, se dejó de lado que la emoción de miedo, considerada también como primaria ante una amenaza, comparte los mismos indicadores y la misma reacción fisiológica que la ira (Mikulincer y Shaver, 2011). Por consiguiente, actualmente hay un vacío teórico en el estudio del miedo como emoción primaria ante las situaciones conflictivas con la pareja romántica.

\subsection{Relación entre Ansiedad e Ira}

4.2.1 Ansiedad e Ira-Rasgo: Este hallazgo va en línea con la base teórica que Diamond (2015) emplea para sus investigaciones. Él explica que quienes presentan altos puntajes en las dimensiones de Evitación o Ansiedad tendrían también una predisposición a la hiperreactividad de los sistemas Nervioso Autónomo y Nervioso Hipotalámico-Pituitario-Adrenocortical o límbico. En la vida cotidiana, esto se entiende como una predisposición de estas personas a experimentar ira con frecuencia (Ira-Rasgo) ante un peligro, y en ocasiones miedo (Kasper, Fauci, Longo, Braunwald, Hauser, y Jameson; 2005; Moscoso, 2000; Izard, 1991, en Muro, 2010).

4.2.2 Ansiedad y Expresión de Ira: Los resultados del presente estudio son congruentes con los hallazgos de Gormley y McNiel (2010).

4.2.2.1. Ansiedad y Control de Ira manifiesta, Ansiedad y Control de Ira Contenida: Aquellos con altos puntajes en la dimensión Ansiedad han desarrollado estrategias para evitar tanto la expresión (Control de Ira Manifiesta) como la vivencia (Control de Ira Suprimida) de ira (Moscoso, 2000). Este funcionamiento replicaría lo planteado por Follingstad, Bradley, Helff, y Laughlin (2002), quienes explican que las personas con altas puntuaciones en la dimensión de apego Ansiedad desarrollarían estrategias de control sobre la propia ira y su expresión, pues preferirían emplear estrategias para controlar las 
emociones de la pareja y así evitar que los abandonen. Por tanto, solamente expresarían su ira cuando dichas estrategias de manipulación no son suficientes.

4.2.2.2 Ansiedad e Ira-Estado: Esto es congruente con diversas investigaciones realizadas con técnicas de recolección de datos diferentes a las empleadas en el presente estudio (Nisebaum y Lopez, 2014; Simpson et al., 1996, en Konishi y Hymel, 2014; Bélanger y Brisebois, 2010). Al respecto, en los estudios de Moscoso (2008) acerca de la ira, los participantes reportan consistentemente no encontrarse molestos al momento de la evaluación, lo cual llevó a este autor a plantear un cuestionario de ira sin la dimensión Ira-Estado (Moscoso, 2008). Es de interés, entonces, observar que cuando esta dimensión de Ira se evalúa junto con la exploración de las dinámicas de Apego, sí se encuentra relación entre ambos constructos.

\subsection{Limitaciones}

A pesar de haber obtenido algunos resultados de interés en un área de investigación que se ha explorado de manera incipiente en la literatura, el presente estudio presenta una principal limitación: la imposibilidad de generalización los resultados.

En específico, para poder participar del estudio, se debía tener acceso a redes sociales y a internet, en un país donde solamente el $40 \%$ de la población cuenta con este tipo de comunicaciones (Adame, Arellano, Pérez, y Oliveros, 2016).

Además, las relaciones de pareja en esta investigación son peculiares por darse en contextos urbanos y entre personas que están culminando/han culminado su educación superior. Para Kocur y Deffenbacher (2014), este tipo de relaciones se caracterizaría por ser más igualitaria que aquellas que se dan en otros contextos.

Por otro lado, el grupo de participantes estuvo conformado, en su mayoría (64\%), por mujeres, lo cual podría influir en los resultados obtenidos en lo que respecta a nivel de expresión de ira. En este caso, es necesario notar que a las mujeres se les suele socializar para evitar el empleo de la agresión cuando se encuentran enojadas (Chaplin, Cole, y Zahn-Waxler, 2005), lo cual incidiría en la manera en que manejan la ira y frustración ante situaciones problemáticas (Sánchez, FernándezBerrocal, Montañés, y Latorre, 2008).
De los resultados de la presente investigación se desprenden recomendaciones para futuros estudios. En primer lugar, para lograr una mayor posibilidad de generalización de resultados, se debe verificar si los hallazgos se mantienen al trabajar con personas de características similares a la del presente estudio, pero con mayor cantidad de participantes (300, como en algunos estudios revisados) en un grupo con distribuciones homogéneas entre varones y mujeres.

Con respecto a los instrumentos, es necesario complementar la recolección de datos con un formato impreso, y así llegar a personas que no cuenten con Internet. Asimismo, se recomienda verificar si los cuestionarios mantienen su adecuación lingüística (Alcázar, Deffenbacher, y Byrne, 2011) en contextos rurales del Perú.

Por otro lado, se propone ampliar el periodo de recolección de datos a 7 meses y así aumentar la proporción de participantes que logró culminar los cuestionarios. De esta manera, se abarcaría, con mayor probabilidad y frecuencia, algunos días feriados y periodos de vacaciones donde los participantes tengan mayor disponibilidad de tiempo.

Si bien los resultados obtenidos aportaron datos empíricos que soporten la hipótesis concerniente a la relación entre Ansiedad e Ira-Rasgo, el hecho que las demás hipótesis no hayan encontrado sustento empírico podría llegar a ser de importancia. Como argumenta Popper (1961, en Kantowitz, Roediger, y Elmes, 2001), la evidencia negativa puede llegar a ser más útil que la positiva. Por ejemplo, de repetirse los presentes hallazgos en la dimensión de apego Evitación, se podría empezar a explorar un funcionamiento diferente de este constructo en nuestro contexto. Como mencionan diversos autores (Zayas y Hazan, 2015; Brassard, Darveau, Péloquin, Lussier, y Shaver, 2014), sería limitado entender a la dimensión de Evitación como un rechazo a la intimidad. En cambio, proponen que en estas personas primaría una necesidad de autoafirmación y el miedo a la deprivación social. Es por ello que la supresión de cualquier manifestación de emoción se entendería, bajo este enfoque, como un aislamiento con miras a que sea la pareja quien reafirme su interés por la relación.

A nivel terapéutico, y de replicarse la relación inversa entre la dimensión de apego Ansiedad con la dimensión Expresión de Ira, sería de utilidad adaptar las intervenciones con este tipo de 
pacientes. Así, como en las intervenciones de López, Rodríguez, Vázquez, y Alcázar (2012), se debería tomar en cuenta que, para estas personas, la prioridad es mantener el vínculo romántico. Por tanto, forzarlos a expresar su ira hacia la pareja activaría en ellos emociones negativas, pues significaría pensar en el rechazo que esto traería en consecuencia.

El presente estudio resalta la importancia de recordar que la vivencia y expresión de emociones, como la ira, se relacionan con factores como el mantenimiento de un vínculo significativo para la persona.

\section{ORCID}

Giovana Violeta Chanllío

Ríos:

https://orcid.org/0000-0002-3487-4115

\section{Contribuciones}

Giovana Violeta Chanllio Rios: Autoría, investigación, conceptualización, recursos, análisis formal, escritura borrador-original, administración del proyecto, recolección de datos, formulación de herramienta digital.

Ana Mercedes Caro Cárdenas: Asesoría de tesis, recomendación de literatura, recursos, revisión del borrador de tesis.

Magaly Suzy Noblega Mayorga: Revisión de pares, recomendación de literatura.

\section{Fuente de financiamiento}

El estudio fue autofinanciado

\section{Conflictos de interés}

La autora declara que no existe conflicto de intereses.

\section{Agradecimientos}

A mis padres, por el apoyo incondicional. A Nabooroo, por la compañía. A toda mi familia.

Agradezco también a mi círculo cercano de amigos, a las personas que respondieron y difundieron el cuestionario virtual, a mi asesora de tesis Ana Caro, a Magaly Noblega por sus sugerencias, a mis asesores de prácticas en el Hospital Larco Herrera, al doctor José Mogrovejo, por acompañarme en esa etapa, a todos mis jefes y profesores que haya tenido alguna vez, a mis alumnos, y a mis pacientes. Sobre todo, agradezco a los autores de las pruebas ML-STAXI y ECR-S, por permitirme digitalizar los cuestionarios, y por brindarme toda la información que, consideraron, podría servirme de ayuda. A Dios.

\section{Referencias}

Adame, V., Arellano, A., Pérez, M., y Oliveros, R. (2016). The Digital Context in Peru. Perú: BBVA Research.

Alcázar, R., Deffenbacher, J., y Byrne, Z. (2011). Assessing the Factor Structure of the Anger Expression Inventory (ML-STAXI) in a Mexican Sample. International Journal of Psychology and Psychological Therapy, 11(2), 307-318.

Alcázar-Olán, R., Deffenbacher, J., Hernández, L., y Jurado, S. (2015). High and Low Trait Anger, Angry Thoughts, and the Recognition of Anger
Problems. Spanish Journal of Psychology, 18(84). doi:10.1017/sjp.2015.84

Adams, G., y McWilliams, L. (2015). Relationships between adult attachment style ratings and sleep disturbances in a nationally representative sample. Journal of Psychosomatic Research, 79(1), 37-42. doi: 10.1016/j.jpsychores.2014.12.017

Alonso-Arbiol, I., Balluerka, N. y Shaver, P. (2007). A Spanish version of the Experiences in Close Relationships (ECR) adult attachment questionnaire. Personal Relationships, 63. doi: 10.1111/j.1475-6811.2006.00141.x 
Anderson, E. (2012). The monogamy gap: men, love and the reality of cheating. Oxford Scholarship Online.

doi: 10.1093/acprof:oso/9780199777921.003.0006

Bardales, G. (2013). Representaciones mentales de apego en un grupo de mujeres que presentan desórdenes alimenticios. Lima: Repositorio de Tesis PUCP.

Bartz, J., Baldwin, M., y Lydon, J. (2015). An Expectancy-Value Approach to Attachment. Bases of Adult Attachment: Linking Brain, Mind and Behavior, 35. doi: 10.1007/978-1-4614-962293

Bélanger, C. y Brisebois, H. (2010). Anger as a moderator of the relationships between attachment, dyadic adjustment, and childhood victimization in physically violent spouses. Europe's Journal of Psychology, 6(1), 14-43. doi: 10.5964/ejop.v6i1.170

Bordignon, N. (2005). El desarrollo psicosocial de Eric Erikson. El diagrama epigenético del adulto. (Spanish). Revista Lasallista De Investigación, 2(2), 50-63.

Bowlby, J. (1973). Attachment and loss, Vol. 2: Separation. New York: Basic Books.

Bowlby, J. (1982). Attachment and loss. Vol. 1: Attachment (2nd Ed.). New York: Basic Books.

Brassard A, Darveau V, Péloquin K, Lussier Y, y Shaver P. (2012). Childhood Sexual Abuse and Intimate Partner Violence in a Clinical Sample of Men: The Mediating Roles of Adult Attachment and Anger Management. Journal of Aggression, Maltreatment \& Trauma, 23(7), 683-704. doi: 10.1080/10926771.2014.933464

Brunner, T. y Spielberger, C. (2009). State-trait anger expression inventory-2 child and adolescent (STAXI-2 C/A). Odessa, FL: Psychological Assessment Resources.

Chaplin, T., Cole, P., y Zahn-Waxler, C. (2005). Parental socialization of emotion expression: Gender differences and relations to child adjustment. Emotion, 5(1), 80-88. doi: $10.1037 / 1528-3542.5 .1 .80$

Chopik, W., Edelstein, R. y Fraley, R. (2013). From the cradle to the grave: Age differences in attachment from early adulthood to old age. Journal of Personality, 81(2), 171-183. doi: 10.1111/j.1467-6494.2012.00793.x

Collins, N. y Feeney, B. (2000). A Safe Haven: An Attachment Theory Perspective on Support Seeking and Caregiving in Intimate Relationships. Journal of Personality y Social Psychology, 78(6), 1053-1073. doi: 10.1037//0022-3514.78.6.1053

Diamond, L. M. (2015). The Biobehavioral Legacy of Early Attachment Relationships for Adult Emotional and Interpersonal Functioning. Bases of Adult Attachment: Linking brain, mind and behavior, 79. doi: 10.1007/978-1-4614-9622-9 5
Feeney, B. y Collins, N. (2001). Predictors of caregiving in adult intimate relationships: An attachment theoretical perspective. Journal of Personality and Social Psychology, 80(6), 972994. doi: 10.1037//0022-3514.80.6.972

Fraley, R. (2010). A brief overview of adult attachment theory and research. Recuperado de https://internal.psychology.illinois.edu/ rcfraley/a ttachment.htm

Follingstad, D., Bradley, R., Helff, C., y Laughlin, J. (2002). A model for predicting dating violence: Anxious attachment, angry temperament, and need for relationship control. Violence And Victims, 17(1), 35-47. doi: 10.1891/vivi.17.1.35.33639

Garrido, L., Guzmán, M., Santelices, M., Vitriol, V., y Baeza, E. (2015). Estudio comparativo de los estilos de apego adulto en un grupo de mujeres con y sin diagnóstico de depresión. Terapia Psicológica, 33(3), 285-295. doi:10.4067/S071848082015000300011

Gormley, B. y McNiel, D. (2010). Adult Attachment Orientations, Depressive Symptoms, Anger, and Self-Directed Aggression by Psychiatric Patients. Cognitive Therapy and Research, 34(3), 272-281. doi: 10.1007/s10608-009-9267-5

Grych, J. y Kinsfogel, K. (2010). Exploring the role of attachment style in the relation between family aggression and abuse in adolescent dating relationships. Journal of Aggression, Maltreatment, and Trauma, 19(6), 624-640. doi: 10.1080/10926771.2010.502068

Guzmán, M., y Trabucco, C. (2014). Estilos de apego y empatía diádica en relaciones de pareja en adultos emergentes. Revista Argentina De Clínica Psicológica, 23(1), 61-69. doi: 10.1111/j.17508606.2007.00016.x.

Hazan, C., y Selçuk, E. (2015). Normative processes in romantic attachment. Bases of adult attachment: Linking brain, mind and behavior, 3-8. New York: Springer. doi: 10.1007/978-1-4614-9622-9_1

Hazan, C., y Shaver, P. (1987). Romantic Love Conceptualized as an Attachment Process. Journal of Personality and Social Psychology, 52(3), 511524. doi: 10.1037/0022-3514.52.3.511

Kantowitz, B., Roediger, H. y Elmes, D. (2001). Psicología experimental. México, D.F. Thomson Learning.

Hernández, R., Fernández, C. y Baptista, P. (2014). Metodología de la investigación. México DF: McGraw-Hill.

Instituto Nacional de Estadística e Informática. (2014). Encuesta Demográfica y de Salud Familiar-ENDES. Lima: INEI.

Instituto Nacional Penitenciario (2015). Informe Estadístico Penitenciario. Lima: Unidad de Estadística INPE.

Kasper, D., Fauci, A., Longo, D., Braunwald, E., Hauser, S., y Jameson, J. (2005). Principios de Medicina Interna. Estados Unidos: Mc Graw Hill. 
Keltner, D. y Lerner, J. (2010). Emotion. The handbook of social psychology, Fifth edition. New York: McGraw Hill.

Kobak, R., Cole, H., Ferenz-Gillies, R., Fleming, W., y Gamble, W. (1993). Attachment and Emotion Regulation during Mother-Teen Problem Solving: A Control Theory Analysis. Child Development, 64(1). doi: 10.1111/j.14678624.1993.tb02906.x

Kocur, J. y Deffenbacher, J. (2014). Anger and Anger's Expression Generally and in Romantic Relationships. Contemporary Family Therapy: An International Journal,36(1), 120-134. doi: 10.1007/s10591-013-9271-5

Konishi, C., y Hymel, S. (2014). An attachment perspective on anger among adolescents. MerrillPalmer Quarterly, 60(1), 53-79. doi: 10.13110/merrpalmquar1982.60.1.0053

Loinaz, I. y Echeburúa, E. (2012). Adult Attachment in Partner-Violent men. Acción Psicológica, 9(1), 33-46. doi: 10.5944/ap.9.1.435

Mikulincer, M. (1998). Attachment working models and the sense of trust: An exploration of interaction goals and affect regulation. Journal of Personality and Social Psychology, 74(5), 12091224. doi: 10.1037/0022-3514.74.5.1209

Mikulincer, M., Shaver, P. R., y Pereg, D. (2003). Attachment theory and affect regulation: The dynamics, development, and cognitive consequences of attachment-related strategies. Motivation and Emotion, 27(2), 77-102. doi: 10.1023/A:1024515519160

Mikulincer, M. y Shaver, P. (2007). Attachment in adulthood: structure, dynamics, and change. New York: Guilford

Mikulincer, M., y Shaver, P. (2011). Attachment, anger, and aggression. Human aggression and violence: Causes, manifestations, and consequences. Washington: American Psychological Association. doi: 10.1037/12346013

Moscoso, M. (2000). Estructura Factorial Del Inventario Multicultural Latinoamericano de la Expresión de la Cólera y la Hostilidad. Revista Latinoamericana de Psicología, 32(2), 321-343

Moscoso, M.S., (2008). La Hostilidad: sus efectos en la salud y medición psicométrica en Latinoamérica. Revista Persona, 11, 75-90

Moscoso, M. (2014). Medición Psicométrica de la expresión de la ira y hostilidad. Revista de Psicología de la Universidad César Vallejo, 16(2).

Mujica, J. (2011). Violaciones sexuales en el Perú 2000-2009. Un informe sobre el estado de la situación. Lima: PROMSEX.

Muris. P., Meesters, C., Morren, M., y Moorman, L. (2004). Anger and hostility in adolescents: Relationships with self-reported attachment style and perceived parental rearing styles. Journal of Psychosomatic Research, 57(3), 257-264. doi: 10.1016/S0022-3999(03)00616-0
Muro, M. (2011). Cólera y acoso escolar en un grupo de adolescentes de un colegio estatal en Lima Metropolitana (Tesis de Licenciatura). Pontificia Universidad Católica del Perú: Lima. Recuperado de http://tesis.pucp.edu.pe/repositorio/handle/123456 $789 / 670$

Nisenbaum, M., y Lopez, F. (2015). Adult attachment orientations and anger expression in romantic relationships: A dyadic analysis. Journal of Counseling Psychology, 62(1), 63-72. doi: 10.1037/cou0000047

Ognibene, T. y Collins, N. (1998). Adult attachment styles, perceived social support and coping strategies. Journal of Social and Personal Relationships, 15(3), $323 . \quad$ doi: 10.1177/0265407598153002

Organización Mundial de la Salud. (2013). Hoja Informativa: Comprender y abordar la violencia contra las mujeres. Violencia infligida por la pareja. Washington DC: OPS.

Oshri, A., Sutton, T., Clay-Warner, J., y Miller J. (2015). Child maltreatment types and risk behaviors: Indirect paths via attachment style and emotion regulation dimensions. Personality and Individual Differences, 73(1), 127-133. doi: 10.1016/j.paid.2014.09.015

Overall, N., Simpson, J., y Struthers, H. (2013). Buffering attachment-related avoidance: Softening emotional and behavioral defenses during conflict discussions. Journal of Personality and Social Psychology, 104(5), 854-871. doi: 10.1037/a0031798

Riva, G., Teruzzi, T., y Anolli, L. (2003). The use of the internet in psychological research: Comparison of online and offline questionnaires. Cyberpsychology and Behavior, 6(1), 73-80. doi:10.1089/109493103321167983

Rholes, W. y Simpson, J. (2004). Adult attachment: Theory, research, and clinical implications. New York: Guilford.

Rholes, W., Simpson, J., y Oriña, M. (1999). Attachment and anger in an anxiety-provoking situation. Journal of Personality and Social Psychology, 76(6), 940-957. doi: 10.1037/00223514.76.6.940

Rospigliosi, S. (2010). Auto-lesiones y factores asociados en un grupo de escolares de Lima Metropolitana (Tesis de grado). Pontificia Universidad Católica del Perú: Lima

Sánchez, M., Fernández-Berrocal, Montañés, J., y Latorre, J. (2008). ¿Es la inteligencia emocional una cuestión de género? Socialización de las competencias emocionales en hombres y mujeres y sus implicaciones. Electronic Journal of Research In Educational Psychology, 6(2), 455474.

Sheinbaum, T., Kwapil, T., Ballespí, S., Mitjavila, M., Chun, C, Silvia, P., y Barrantes-Vidal, N. (2015). Attachment style predicts affect, cognitive 
appraisals, and social functioning in daily life. Frontiers in Psychology, 6, 296. doi:10.3389/fpsyg.2015.00296

Secretaría Nacional de la Juventud. (2015). Informe Nacional de las Juventudes 2015. SENAJU: Lima Spielberger, C. (1985). Anxiety, cognition and affect. Anxiety and anxiety disorders, 351-376. Hilsdale: Erlbaum.

Spielberger, C. (1988). State-Trait-AngerExpression-Inventory. Consulting Psychologist Press: Palo Alto.

Spielberger, C. y Reheiser, E. (2009). Assessment of Emotions: Anxiety, Anger, Depression, and Curiosity. Applied Psychology: Health and WellBeing, 1(3), 271-302. doi: 10.1111/j.17580854.2009.01017.x

Stevens, F. (2014). Affect Regulation Styles in Avoidant and Anxious Attachment. Individual Differences Research, 12(3), 123-130.

Trnka, R. y Stuchlóková, I. (2011). Anger coping strategies and Anger regulation. Re-Constructing
Emotional Spaces: From Experience to Regulation. Czech Science Foundation: República Checa

Varnhagen, C., Gushta, M., Daniels, J., Peters, T., Parmar, N., Law, D., Hirsch, R., Takach, B. S., y Johnson, T. (2005). How informed is online informed consent? Ethics \& Behavior, 15(1), 37 48. doi: 10.1207/s15327019eb1501_3

Wilson, T.; Aronson, E. y Carlsmith, K. (2010). The art of laboratory experimentation.

Zayas, V., Günaydin, G., y Shoda, Y. (2015). From an unknown other to an attachment figure: How do mental representations change with attachment formation? Bases of Adult Attachment: Linking Brain, Mind and Behavior. doi: 10.1007/978-14614-9622-9_8

Zimmermann, P. (2004). Attachment representations and characteristics of friendship relations during adolescence. Journal of Experimental Child Psychology, 88(1), 83-101. doi:10.1016/j.jecp.2004.02.002 